\title{
贯穿论文式实验报告的综合能力培养教学实践
}

田东亮 ${ }^{*}$ 杨青林，刘克松，朱英

北京航空航天大学化学学院, 北京 100191

摘要: 加强实验教学是培养学生科学素养和综合创新能力的重要途径。基于 “两性一度” 的课程教学要求, 针对目 前实验教学中撰写实验报告存在的问题, 以物理化学实验课程为例, 进行了教学改革与实践。采取贯穿科研论文式 实验报告撰写各环节的科学素养和能力培养模式, 该论文式实验报告有利于学生整体把握, 提出问题、分析问题和 总结, 实验报告的各个部分与实际教学环节相统一, 取得了满意的效果。充分发挥了教师主导和学生主体的协同优 势, 激发了学生学习的积极性和主观能动性, 有利于培养德智体美劳全面发展的高素质人才。

关键词: 物理化学实验; 科研论文; 实验报告; 科学素养; 创新能力

中图分类号: G64; O6

\section{Teaching Practice of Innovation Ability Training throughout the Scientific Paper-Type Experimental Report}

\author{
Dongliang Tian *, Qinglin Yang, Kesong Liu, Ying Zhu \\ School of Chemistry, Beihang University, Beijing 100191, China.
}

\begin{abstract}
Strengthening laboratory teaching is an important way to cultivate students' scientific literacy and comprehensive innovation ability. Taking physical chemistry laboratory as an example, the teaching reform and practice were carried out to solve the problems of writing experimental report. A research paper type experimental report writing format was adopted for undergraduate course teaching, which is helpful for students to understand the whole picture of the experiment, put forward questions, analyze questions and summarize the experiment. The cultivation of students' scientific literacy and comprehensive innovation ability has been achieved throughout each part of the experimental report, which is unified with the actual teaching link. It gives the full play to the synergetic advantages of teacher-led and student-subject, stimulates students' learning enthusiasm and subjective initiative, and is conducive to the cultivation of high-quality talents with comprehensive development of characteristics including morality, intelligence, physique, aesthetics and labor.
\end{abstract}

Key Words: Physical chemistry laboratory; Research paper; Experimental report; Scientific quality; Innovation ability

新形势下, 具有创新、创业、创造能力的高素质人才是解决国家发展重大战略需求的关键。建 立和健全人才培养机制和创新教育课程体系是培养扎根中国大地的一流人才的重要保障。化学作为 最重要的基础科学之一, 在人类生存和社会发展进步中具有重要意义。实验是化学的基础和生命, 在 揭示化学本质中起到了重要的作用。实验教学是提高学生动手能力、培养学生科学素质和综合创新

收稿: 2020-08-01; 录用: 2020-09-14; 网络发表: 2020-10-09

“通讯作者, Email: tiandl@buaa.edu.cn

基金资助: 北京航空航天大学一流本科课程(物理化学实验); 北京航空航天大学教改重点项目( “大学化学” 课程实验考核方式的改革与实践) 


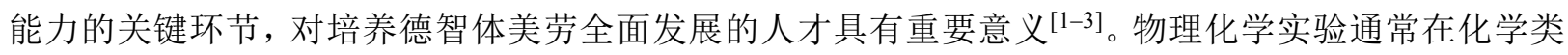
相关专业本科生高年级开设, 是建立在 “四大化学” 中无机化学、分析化学、有机化学知识及其实 验基础上理论联系实际应用的高阶课程 ${ }^{[4-7]}$ 。实验课程的开设中最重要的两部分就是实验操作和实验 报告撰写。冊庸置疑, 实验操作是培养学生动手能力和科学素养的重要过程, 而实验报告的撰写不 仅是学生对实验教学过程的观察记录、分析讨论、归纳总结和整体把握, 而且是强化实验操作能力、 加强学生自主分析并解决复杂问题的科学素质和综合创新能力培养的重要环节 ${ }^{[8-11]}$ 。同时, 实验报 告的撰写通常是教师了解学生实验掌握情况并进行实验成绩评定的重要途径。

但是, 在教学过程中, 发现学生对目前实验教学中普遍采用的实验报告敷衍了事、照搬照抄, 甚至存在弄虚作假的现象, 这种流于形式的实验报告区分度低, 难以实现学生科学素养和创新能力 培养的课程目标 ${ }^{[12-15]}$ 。针对这一实际问题, 我校结合化学类专业学生特点, 进行了科研论文式实验 报告撰写的教学实践, 激发了学生的学习兴趣, 提高了学生的积极性和主动性, 强化了贯穿整个实 验教学过程的学生科学素质和综合创新能力培养, 获得了满意的效果。

\section{1 我校物理化学实验报告改革情况}

我校物理化学实验课程是面向大二、大三年级化学、材料、环境三个学院四个专业开设, 每年 选课人数为 240 人左右。分为物理化学实验 $\mathrm{A}$ 和物理化学 $\mathrm{B}$ 实验 2 类。其中, 物理化学实验 $\mathrm{A}$ 是针对化 学、应用化学专业独立开设的一门课程, 分春秋两个学期开设, 总学时为 112 。实验教学中采取了研 究型教学模式, 得到了较好的效果。针对实验报告撰写中普遍存在的问题, 结合我校具体情况, 我 们进行了科研论文式实验报告撰写的教学实践。通过将科研论文式实验报告与实验教学的各个环节 紧密结合与统一, 加强了学生整体掌握实验的知识原理、操作技能、应用方向及解决问题的一般方 法。充分发挥了教师为主导和学生为主体的协同优势, 激发了学生的学习兴趣, 提高了学生参与的 积极性和主动性, 强化了实验教学过程各环节对学生科学素质和综合创新能力培养的作用和效果。

\section{2 科研论文式实验报告教学实践}

实验报告的撰写是实验教学中最重要的环节之一。根据课前调查结果, 学生已经普遍适应了其 他课程所延续且习惯使用的常规实验报告撰写方式(见表1), 但是这种报告很多部分与实验教材/讲义 内容基本一致, 经过长期的学习与实践, 学生就习惯了把内容从教材/讲义 “搬运” 到实验报告上的 撰写方法, 基本不用动脑, 也谈不上总结概括, 实验报告千篇一律, 学生也不重视, 存在严重的照 抄现象, 不利于学生科学素养的培养, 失去了实验报告撰写的意义。针对这一情况, 我校对物理化 学实验的实验报告撰写进行了改革, 所有实验都要采用科研论文式实验报告。注重学生的价值塑造, 理论实践知识的融合, 培养学生观察、分析、概括总结及全局把握的能力。

表1 常规实验报告格式及要求

\begin{tabular}{clll}
\hline 序号 & 部分名称 & & \multicolumn{1}{c}{ 要求 } \\
\hline 1 & 实验名称 & 与讲义一致 & \\
2 & 实验目的 & 与讲义一培养目标 \\
3 & 实验原理 & 简明扼要 & \\
4 & 实验步骤 & 主要仪器、药品, 仪器应注明型号; 简述实验方法及步骤 & 概括总结、表达的能力 \\
5 & 数据/现象记录 & 正确、客观、完整地记录现象及数据 & 观察、记录的能力, 严谨求实态度 \\
6 & 数据处理 & 将原实验记录重新制表抄写、数据计算、误差计算(与文献值相比较) & 分析与总结表达能力 \\
7 & 结果或结论 & 列出实验结果 & 总结表达的能力 \\
附加材料 & 注意事项 & 写出实验中注意事项 & 加深对实验中关键点的理解和掌握 \\
& 收获与体会 & 根据自己情况, 可不写 & 实验的整体回顾与理解 \\
\hline
\end{tabular}




\section{1 转变观念, 学以致用的科研论文式报告}

在教学过程中, 首先要转变学生观念, 在首次上课时就和学生讲解科研论文式实验报告的重要 性, 强调与常规实验报告的区别, 并给出科研论文式实验报告格式要求(见表2)。科研论文式实验报 告不仅仅是实验的整体理解, 更是普适性方法的学习。科研论文式实验报告从概括总结, 到提出问 题、分析问题、解决问题的设想提出, 到详细方案的设计与实施, 再到实验数据的记录、分析、总 结与讨论, 最后给出切身体会和建设性意见及建议, 是创造性思想的载体, 各个过程无不渗透着能 力的培养和综合素质的提高。让学生从思想上重视起来, 激发学生的求知欲, 努力提高自身能力的 主动性。

表2 科研论文式实验报告格式及要求

\begin{tabular}{|c|c|c|c|}
\hline 序号 & 部分名称 & 要求 & 能力培养目标 \\
\hline 1 & 题目 & 根据具体实验内容、方法等进行总结 & 准确、简洁、清楚地概括总结、表达的能力 \\
\hline 2 & 作者 & 学生自己和合作者 & 团结协作与严谨求实的态度 \\
\hline 3 & 摘要 & $\begin{array}{l}\text { 整体概括实验, 背景、方法、研究对象、重要 } \\
\text { 结果和主要结论 }\end{array}$ & $\begin{array}{l}\text { 理清整体实验, 培养提出问题、分析问题、解决 } \\
\text { 问题, 及概括总结表达能力 }\end{array}$ \\
\hline 4 & 关键词 & 选择反映论文主题内容的词 & 关键内容表达 \\
\hline 5 & 前言介绍 & $\begin{array}{l}\text { 给出研究工作背景, 前人做了哪些工作, 存在 } \\
\text { 的不足及遗留问题, 本实验希望通过什么理论 } \\
\text { 方法和实验基础解决什么问题, 该问题的解决 } \\
\text { 有什么作用和意义 }\end{array}$ & $\begin{array}{l}\text { 查阅文献, 提出问题、分析并解决问题的总结表 } \\
\text { 达能力及创新创业能力 }\end{array}$ \\
\hline 6 & 实验部分 & $\begin{array}{l}\text { 简述主要仪器、药品, 仪器应注明型号, 实验 } \\
\text { 方法及步骤 }\end{array}$ & 理清实验操作思路及注意事项, 概括总结的能力 \\
\hline 7 & 结果讨论 & $\begin{array}{l}\text { 利用原始数据直接作图, 图文并茂给出计算、 } \\
\text { 分析、比较、讨论和误差比较等详细描述过程 }\end{array}$ & 分析总结表达能力、科学素养及创新创业能力 \\
\hline 8 & 结论 & 给出直接结论、间接结论和推论 & 科学意识、总结表达能力及创新创业能力 \\
\hline 9 & 参考文献 & 引用前人已发表的文献中观点、数据和材料等 & 尊重他人成果, 客观严谨的科学态度 \\
\hline 附加材料 & 体会和建议 & 必须认真写出实验体会和实验改进建议 & 提出合理的建设性意见和建议 \\
\hline & 原始数据 & 正确、客观、完整地记录现象及数据 & 科学严谨的态度 \\
\hline
\end{tabular}

\section{2 层层递进式培养科研论文报告撰写能力}

鉴于大部分学生还未涉及科研工作, 对科研论文的概念、格式和要求都不尽熟悉, 在最初几次 撰写时给学生提供科研论文模板, 以及前面学生的优秀实验报告作为参考, 逐渐熟悉科研式实验报 告, 最终达到实验中科学素质和综合创新能力的培养。目前, 我们开设的实验有经典优化实验 4 个), 经典+设计实验 $(6$ 个), 设计实验 $(4$ 个)和综合实验 $(2$ 个)四部分。对于经典优化实验部分, 由于其步骤 内容已经基本确定, 设计成分较少, 我们将其作为科研论文式实验报告的起步阶段, 为学生提供标 准的论文模板, 比如往届同学较好的科研论文式实验报告作参考, 要求学生熟悉科研式论文撰写格 式; 在经典+设计实验阶段, 有部分内容需要设计, 学生可以在熟悉格式的基础上进行部分发挥; 设 计实验阶段, 实验中步骤内容等设计程度继续加大, 学生可以加强发挥; 在综合实验阶段, 综合设 计性强, 可以实现学生自由发挥的科研式论文撰写。通过层层递进的实验内容设计, 实现层层递进 的科研式论文报告撰写能力培养(如图1)。

\section{3 紧密联系教学各环节, 培养综合创新能力}

实验报告是在实验教学过程中, 再次对实验课程设计所有环节的回顾和总结。实验报告的撰写 情况直接反映教学实施过程中教师的教学质量和学生的学习效果。因此, 紧扣教学各环节提高教学 
质量, 加强培养学生的操作能力和分析总结能力是实现教学培养目标的关键。在科研论文式实验报 告的撰写中, 每部分的能力培养目标都与不同教学环节相对应, 环环相扣, 紧密结合, 可以实现贯 穿教学各环节的能力培养。通过充分发挥教师教的主导和学生学的主体的协同优势, 将课程与科研 论文式实验报告训练结合, 实现认知 $\rightarrow$ 应用 $\rightarrow$ 拓展 $\rightarrow$ 创新能力的提升, 培养解决问题的逻辑思维方 法, 总结表达和创新能力, 突出课程的高阶性、创新性和挑战度, 提升学生的综合创新能力(如图2)。

层层递进、阶梯式上升的实验设计

\begin{tabular}{|c|c|c|c|}
\hline $\begin{array}{l}\text { 经典优化 (4个) } \\
\text { 结合学科特色 } \\
\text { 联系实际优化 }\end{array}$ & $\begin{array}{c}\text { 经典+设计 (6个) } \\
\text { 增加未知内容 } \\
\text { 激发积极性 }\end{array}$ & $\begin{array}{c}\text { 设计 (4个) } \\
\text { 针对选取实验 } \\
\text { 独立完成设计 }\end{array}$ & $\begin{array}{c}\text { 综合 (2个) } \\
\text { 针对特色研究 } \\
\text { 加强综合创新 }\end{array}$ \\
\hline$\square$ & ए & ए & 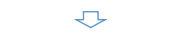 \\
\hline $\begin{array}{c}\text { 熟悉格式 } \\
\text { 结合实验内容 } \\
\text { 熟悉基本撰写 }\end{array}$ & $\begin{array}{c}\text { 部分发挥 } \\
\text { 根据设计增加 } \\
\text { 撰写自由度 }\end{array}$ & $\begin{array}{c}\text { 充分发挥 } \\
\text { 框架下独立 } \\
\text { 设计与撰写 }\end{array}$ & $\begin{array}{c}\text { 自由发挥 } \\
\text { 依据高阶实验 } \\
\text { 加强创新 }\end{array}$ \\
\hline
\end{tabular}

渐进式科研论文报告撰写能力培养

图1 层层递进式的科研论文撰写能力培养

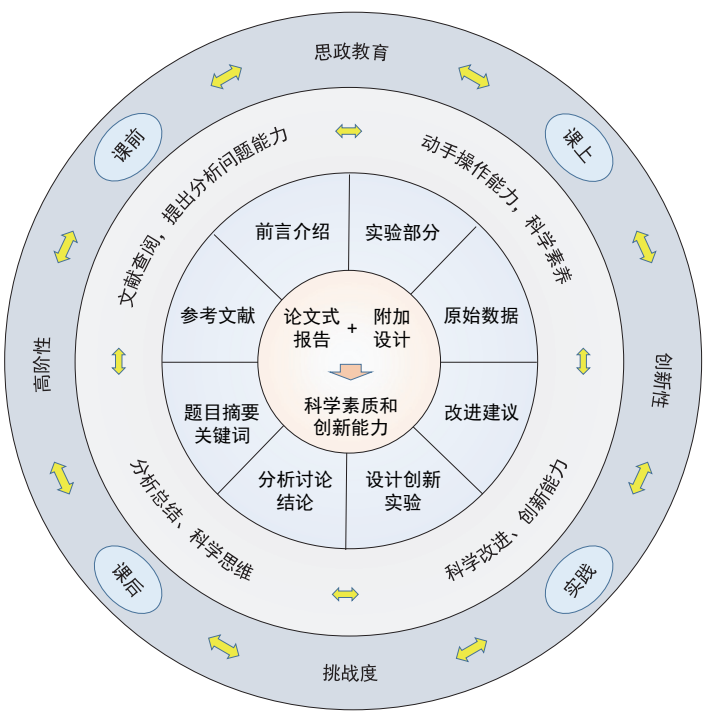

图2 贯穿教学各环节提升学生科学素质和创新能力的科研论文式实验报告设计

\subsection{1 课前预习与前言介绍相统一}

课前预习是物理化学实验课非常重要的环节, 学生通过阅读教师提供的实验讲义及文献资料, 了解实验的内容与目的、原理和方法等, 其中最关键的部分是掌握实验的研究背景, 通过查阅相关 文献, 便可以知道实验研究所处的理论基础和当时的实验条件。通过这一过程的学习, 学生不仅知 道了实验的重要性, 当时所具有的知识背景, 当时存在的问题, 解决问题所用方法的先进性和对现 在知识结构和化学的影响, 更重要的是, 从中学会了问题解决的一般方法和思路。这样就为科研论 文式实验报告的前沿介绍内容提供了好的素材。一般情况下, 学生调研充分, 认识深刻的在前言介 绍中都会有相应关键内容的体现。比如研究的重要性, 当时存在的问题, 当时解决问题的设计思路 等等。以燃烧热的测定实验为例, 在课前学生学习了燃烧热的讲义及相关资料后, 会查阅新资料, 了解燃烧热的定义、测试方法和应用范围, 从而知道生活中食品热量、生产中的燃料热量和航空航 天高能燃料的选择和使用, 从而将该部分内容总结反映到科研论文式实验报告的前言部分, 这样教 
师很容易就判断出学生的预习资料查阅和总结情况。加强分析、综合和判断的能力, 体现课程的 “高 阶性”。

\subsection{2 课中实验操作及实验数据相统一}

在课堂教学过程中, 学生按照课前预习(给定步骤或设计步骤)进行实验操作, 并得到相应的实 验数据, 数据要边做边记录, 原始数据不允许私自涂改, 必须按照科学规范进行修改, 最终得到的 原始数据要放到实验报告后面一起交, 培养学生严谨求实的科学态度和科学素养。反映在科研论文 式实验报告中, 实验操作部分要简明扼要, 能概括出具体的思路即可, 让学生加深理清实验思路和 流程, 谨防记住了每步的具体步骤而没有建立逻辑框架。同时, 实验中的操作注意事项和改进创新 内容也会反映到具体实验报告中, 实现理论实践相结合, 实践有利于创新能力的培养。

\subsection{3 课后分析讨论与逻辑思维相统一}

对学生来说, 课后最主要的工作就是完成实验报告。在科研论文式实验报告的撰写中, 实际上 是对实验过程的再现、分析与讨论, 要求学生学会总结凝练内容。如, 题目、摘要、结果与讨论和 结论等。下面就其中几个主要部分简要说明:

对于题目, 要根据具体实验内容、方法等进行总结, 例如在燃烧热的测定中, 采用氧弹量热计 测定了棉花、面粉或航空燃料的燃烧热, 有的学生就将题目总结为 “氧弹量热法测定航空煤油的热 值” “氧弹量热法测定棉花的热值”，等等，锻炼了准确、简洁、清楚地概括总结、表达的能力。

摘要则反映的是对整个实验的总结, 需要概括实验背景、方法、研究对象、重要结果和主要结 论等, 最能体现学生对整个实验是否理清和掌握, 同时培养学生提出问题、分析问题、解决问题, 及概括总结表达能力。

前言介绍反映的是课前的文献调研和总结描述。

实验部分需要总结实验涉及的主要仪器、药品等, 简述实验方法及步骤, 也可以在结果与讨论 部分一并描述。这两部分在课前预习中已经基本完成，稍加组织语言即可。

结果与讨论是实验报告中的重点, 需要将原始数据直接作图、校正、分析与讨论, 该内容注重 图文并茂讲述, 夹叙夹议, 比较和讨论结果, 最终得出合理的结论。通过这一训练过程, 学会使用 Origin、Excel、Matlab等专业软件的使用方法, 常规表达的一般方法和基本常识, 锻炼学生分析总结 表达能力和科学素养。

结论中要给出直接结论、间接结论和推论, 培养学生的理论联系实际并进行扩展的能力, 科学 意识、总结表达能力及创新创业能力。

通过科研论文式实验报告这部分的训练, 学生深层次明确这个实验设立的原因。

\subsection{4 课后设计实践与科学创新相统一}

在实验讲义的最后一部分, 我们安排了设计实验, 这部分是基于学生对实验内容的深层次理解 和掌握, 进行基于实验内容的进一步拓展, 即完成设计性实验, 要求学生给出设计方案, 包括仪器、 步骤和测试原理等主要设计依据, 学生对这部分实验普遍认可, 认为 “不仅加深了原理的理解, 也 提高了解决复杂实际问题的能力, 创新思维和创新能力”。

\subsection{5 课后总结反馈与教学相长相统一}

体会和建议是我们要求科研论文式实验报告的另一重点附加内容, 这也是要求学生必须认真写 出的一部分, 要求学生提出合理的建设性意见和建议。一方面可以提升学生的综合创新创业能力, 实现课程培养目标。另一方面, 还可以进行教学实验的优化与改进。同时, 实验报告通过电子邮件 进行收发, 采用电子化批改过程, 极大地提高了收发及批改的交流效率。我们根据前期统计, 一般 情况下, 学生在实验课后 $2-3$ 天内完成报告的比例达 $60 \%$ 以上, 5 天提交达 $80 \%$ 以上, 而在截止日期附 近的只有不到 $20 \%$ 的比例, 这样大部分学生 $(80 \%$ 以上)可以在实验结束后一周内就得到批改后的报 告, 而且学生交报告早, 就可以早得到反馈建议, 可以在一定程度上减少学生由于长时间等待遗忘 的问题。 


\section{4 科研式实验报告成绩评价}

物理化学实验考核注重过程考核, 加强了学生靠自己努力获得成绩提高的成就感, 激发和提高 了学生的主动性。在物理化学实验期末成绩中, 科研式实验报告成绩占 $40 \%$, 其余 $60 \%$ 由平时成绩 $20 \%$ +操作 $20 \%$ +期末设计 $20 \%$ 构成, 其评分标准如表3所示。以此为基础, 加强了学生的动手能力, 强化了严谨意识和科学素养, 提升了贯穿整个实验教学过程的能力培养, 提高了物理化学实验课的 教学质量和效果, 发挥了教师 “导”与学生 “学” 的协同优势。

\section{表3＼cjkstart科研论文式实验报告培养目标及评分标准}

\begin{tabular}{|c|c|c|c|}
\hline 序号 & 部分名称 & 培养目标 & 评分标准 \\
\hline 1 & 题目 & $\begin{array}{l}\text { 准确、简洁、清楚地概括总结、表达的 } \\
\text { 能力 }\end{array}$ & 5分。要求正确无误, 能反映实验方法及实验研究对象及内容 \\
\hline 2 & 作者 & 团结协作与严谨求实的态度 & 虚构、抄袭的直接零分 \\
\hline 3 & 摘要 & $\begin{array}{l}\text { 理清整体实验, 培养提出问题、分析问 } \\
\text { 题、解决问题, 及概括总结表达能力 }\end{array}$ & $\begin{array}{l}\text { 15分。描述实验背景、方法、研究对象、重要结果和主要结论, } \\
\text { 缺一条扣 } 3 \text { 分 }\end{array}$ \\
\hline 4 & 关键词 & 关键内容表达 & 5分。要求正确无误, 具有代表性, 能反映论文主题内容的词 \\
\hline 5 & 前言介绍 & $\begin{array}{l}\text { 查阅文献, 提出问题、分析并解决问题 } \\
\text { 的总结表达能力及创新创业能力 }\end{array}$ & $\begin{array}{l}20 \text { 分。给出研究背景, 存在的不足及问题, 本实验方法和实验 } \\
\text { 基础, 该问题解决的意义, 缺一条扣 } 4 \text { 分 }\end{array}$ \\
\hline 6 & 实验部分 & $\begin{array}{l}\text { 理清实验操作思路及注意事项, 概括总 } \\
\text { 结的能力 }\end{array}$ & $\begin{array}{l}15 \text { 分。简述主要仪器、药品, 仪器应注明型号, 实验方法及步 } \\
\text { 骤。要求内容清楚, 步骤简洁扼要, 顺序正确。实验内容及步 } \\
\text { 骤应简洁, 直接照抄讲义者扣 } 10 \text { 分; 实验顺序错乱者, 扣 } 3-5 \\
\text { 分; 实验过程描述错误, 每一条扣 } 3-5 \text { 分 }\end{array}$ \\
\hline 7 & 结果讨论 & 分析总结表达能力、科学素养 & $\begin{array}{l}20 \text { 分。利用原始数据直接作图, 给出计算、分析、讨论和误差 } \\
\text { 比较等详细描述过程, 图文并茂, 叙述简洁完整、重点突出, } \\
\text { 依据正确。若数据直接抄写, 没有分析者, 扣 } 10 \text { 分; 数据计算 } \\
\text { 要有过程, 缺少者扣 } 3-5 \text { 分; 实验者没有对结果进行分析, 扣 } 3- \\
5 \text { 分 }\end{array}$ \\
\hline 8 & 结论 & 科学意识、总结表达能力 & 10 分。给出直接结论和推论, 缺一扣 5 分 \\
\hline 9 & 参考文献 & 尊重他人成果, 客观严谨的科学态度 & 5分。引用前人已发表的文献中观点、数据和材料等 \\
\hline $\begin{array}{l}\text { 附加 } \\
\text { 材料 }\end{array}$ & 体会和建议 & $\begin{array}{l}\text { 提出合理的建设性意见和建议, 创新创 } \\
\text { 业能力 }\end{array}$ & 5分。必须认真写出实验体会, 鼓励写出实验改进建议 \\
\hline & 原始数据 & 科学严谨的态度和创新意识 & $\begin{array}{l}\text { 教师签字的实验记录, 没有此页者实验报告为不合格报告, 需 } \\
\text { 要重新实验), 伪造数据或抄录他人数据者实验报告为零分! }\end{array}$ \\
\hline 附加 & & & 加分项目: 能指出实验内容外影响因素, 并能够其进行细致讨 \\
\hline 项目 & & & $\begin{array}{l}\text { 论者, 加1-5分; 能给出实验合理化建议的, 加5-15分, 实验报 } \\
\text { 告总分不超过 } 100 \text { 分 }\end{array}$ \\
\hline
\end{tabular}

\section{5 同行及学生反馈}

通过科研论文式实验报告的实施, 学生普遍认可我们采取的教学改革, 所有学生都按照要求完 成, 其中 $85 \%$ 的学生能认真对待完成, 50\%完成的较好, 30\%的学生完成的非常好。学生最初接触这 个报告是难以进入状态的, 特别是第一次, 绝大多数学生是不知所措的, 这时我们必须加强与学生 的沟通, 需要做学生工作, 采取了鼓励的措施: 第一, 通过微信群和课程中心(学校课程网站)提供了 论文模板和上届学生写得比较好的作为示范, 这样学生的压力能减轻不少; 第二, 学生提交报告后, 教师对每位学生都进行针对性批改和讨论交流, 提出概念意见和建议; 第三, 每次实验课前对前一 
次实验存在的共性问题进行说明, 个别同学写得好的地方进行展示, 让大家互相学习优点。通过 2-3次实验后, 学生对报告模式基本熟悉, 后面的实验就能顺利完成。第一学期期末的问卷调查显示, $95 \%$ 以上的学生都肯定了论文式报告对个人能力和科学思维培养方面的重要性, $85 \%$ 以上的学生愿 意坚持完成。

从学生论文式报告的撰写来看, 每位学生的报告在语言表达、文字及符号(包括脚标)出错率、图 文的美观性等方面, 逐渐加强, 而且在设计及讨论部分能多角度分析。学生每次的论文式报告体会 建议及期末问卷调查显示, 学生普遍欢迎这一模式, 表现在报告撰写熟练, 认为自己的分析解决问 题能力得到了提升, 而且在最近一学期的学生评教中获得93分, 远高于学校平均分 90.44 分。

通过体会和建议及期末问卷调查, 学生对课程给出了肯定评价, 认为 “虽然起步上手难, 但是 可以进一步发现实验中存在的问题, 真正实现总结、绘图、分析和组织、逻辑思维分析和创新思维 等综合创新能力的培养, 真正实现由 “知识” 向 ‘方法” 学习的转变......” , “撰写报告有进步, 翻天覆地的变化……收获大！......” , “值得在其他课程中推广”, 等等。进一步说明了我们采取 科研论文式实验报告撰写有利于加强对学生综合能力的提升。

论文式实验报告的改革经验也得到了院领导和校督导组老师的认可, 并拟在大学化学实验的教 学中选取1-2个实验进行推广。

\section{3 结语}

针对实验教学过程中实验报告存在的问题, 结合化学类专业学生特点, 进行了科研论文式实验 报告撰写的教学实践。通过要求学生按照科研论文格式撰写实验报告, 加强学生对实验教学过程的 整体把握, 即再次从背景出发, 明确实验原理和方法设计实验方案, 根据操作获得实验数据分析、 归纳、总结讨论、得出结论, 同时给出体会和改进建议, 进一步深入理解实验的设计原理和操作方 法及注意事项, 从中学习解决问题的一般方法。结果表明, 极大提高了学生的积极性和主动性, 形 成了严谨求实的科学态度, 强化了综合创新能力培养, 获得了满意的效果, 为其他实验课程教学提 供了很好的范例。

\section{参 考 文 献}

[1] 张树永, 侯文华, 才国旺. 大学化学, 2017, 32 (2), 9.

[2] 张树永, 朱亚先, 张剑荣. 大学化学, 2018, 33 (10), 1.

[3] 张霞, 李伟, 王育红. 实验技术与管理, 2007, 24 (2), 123.

[4] 夏春兰, 邓立志, 刘欲文, 黄驰. 实验室研究与探索, 2013, 32 (8), 181.

[5] 郭婷, 孟涛, 方伊, 童志平, 舒学涁. 实验室研究与探索, 2015, 34 (5), 138.

[6] 訾学红, 陈永宝, 张红光, 刘淑珍, 王小逸. 中国校外教育, 2013, No. 1, 79 .

[7] 王丽莎, 曹晓燕, 姬泓嵬, 包木太, 李先国. 实验科学与技术, 2017, 15 (5), 103.

[8] 张国艳, 金为群, 王岗. 实验室研究与探索, 2013, 32 (6), 329.

[9] 刘钢, 朱万春. 化学教育, 2016, 37 (20), 31 .

[10] 吴岩. 中国大学教学, 2020, No. 4, 4 .

[11] 强胜. 中国大学教学, 2019, No. 11, 29.

[12] 姚青, 张继荣, 李燕, 李建宁, 张茜, 赵薇, 裴秀英. 陕西教育: 高教版, 2011, No. 6, 105.

[13] 李江滨, 蔡梦珊, 李育超, 侯敢. 教育教学论坛, 2013, No. 46, 188.

[14] 莫润阳, 王成会, 张明铎. 物理通报, 2017, No. 12, 70.

[15] 徐强, 杨玲珍. 实验室科学, 2019, 22 (4), 128. 